\title{
Diversité, ordre et unité du vivant dans les savoirs populaires
}

\author{
CLAUdine FriedBerg
}

\author{
On ne peut pas, comme on le fait de plus en plus fréquemment, en appeler aux \\ savoirs populaires pour la gestion de la biodiversité ou le développement durable, \\ sans savoir exactement de quoi il s'agit. Voici un effort de clarification et une prise \\ de position qui viennent es éthnosciences et qui s'adressent tant aux écologues \\ qu'aux divers spécialistes des sciences du développement.
}

\section{Introduction}

Les savoirs populaires n'ont jamais été autant sollicités par les différentes disciplines qui traitent de l'environnement alors que dans le même temps jamais les idées à leur sujet nont été aussi confuses. La raison en est sans doute que ces savoirs ont été l'objet de curiosité depuis très longtemps et que l'on mélange des faits recueillis à des époques et dans des perspectives diverses.

Moqués par les uns, parés de toutes les vertus par les autres, ils sont devenus un enjeu politique et économique maintenant que l'affirmation d'un lien entre diversité biologique et diversité culturelle est une revendication des minorités vivant dans des zones économiquement marginalisées ; cette revendication figure même, au moins dans les textes, dans les conventions internationales.

Cependant trop souvent les recherches sur les savoirs sont improvisées et on oublie qu'ils sont l'objet d'études spécialisées de la part d'anthropologues, en particulier dans le cadre de l'ethnoscience. D'une façon générale, on se contente de relever les usages des plantes et des animaux ou l'on envisage la façon dont les savoirs et les savoir-faire liés aux pratiques sur la nature interviennent dans la gestion de la biodiversité, mais ces études sont menées d'un point de vue extérieur à celui des acteurs et sans se préoccuper de la façon dont ces savoirs s'inscrivent dans la conception que ces derniers se font du fonctionnement du monde.

Ceci est particulièrement gênant quand il s'agit de sociétés non-modernes, vivant sur un mode sociocosmique, c'est-à-dire où les relations avec les autres êtres vivants sont vécues à l'intérieur des relations sociales et où il n'existe pas de coupure nature/culture. II faut alors examiner les savoirs liés aux plantes et aux animaux dans le cadre de l'ensemble de ces relations. Mais ceci vaut également pour les sociétés dites - modernes - dans lesquelles subsistent des aspects non-modernes ${ }^{1}$.

Le malentendu est tel que l'on aboutit, dans les écrits de nombre de ceux qui se penchent sur l'avenir de l'environnement * naturel = de la planète, à des CLAUDine FriEDberG attitudes contradictoires. D'un côté, on constate que Laboratoire d'ethnobioles savoirs populaires prennent en compte la diversité logie-biogéographie. des êtres vivants, en particulier en recueillant les Muséum national usages auxquels ils donnent lieu. De l'autre, on nie d'histoire naturelle, cette reconnaissance de la diversité en déclarant qu'il 57, rue Cuvier, ne faudrait pas revenir, sous prétexte de vouloir établir de nouvelles relations à la nature, à l'ancienne confusion qui aurait existé dans le passé et qui existerait encore dans les cultures non-modernes, entre les hommes et les autres êtres vivants ${ }^{2}$. Cette confusion est associée au fait que, dans ces sociétés, on prête
75231 Paris cedex 05 Claudine Friedberg est directeur de l'Ura 882 du CNRS
Abstract: Diversity, order and unity of living beings in popular knowledge

At a time when popular knowledge about managing the environment is appealed to more and more frequently, it appears important to understand how and by what organising principles it is enacted. For the animal and vegetal environment, it is essential to understand up to what degree of diversity and discretion one organism can be identified, and what type of classification can be applied. This is precisely what is examined here, with the help of examples taken from diverse societies, including France, and concerning the vegetal world. Our objective here is to show how representations of the latter are in relation to the plant features, to Man's own cognitive processes, and to the role plants are performing in those societies. The recognition and classification of the diversity of living beings correspond to two levels of knowledge organisation that can be apprehended from designation terms. A third level does exist, encompassing all living beings, including human, in the functioning of each society. It concerns not only individuals, but also the elements that they are made up of, that condition their birth and growth, in the course of their existence, and at the moment of their death.

The example presented here concerns one non-modern society, yet the reasoning principle at stake is just as valid for the non-modern aspects of our own societies. The ideas that one society makes up about its own functioning and about the whole universe give meaning to the knowledge. It is necessary to take this into account when we take action in managing the environment.
${ }^{1}$ Nous ne pouvons ici nous étendre sur ce qu'est la modernité. Précisons cependant qu'il ne faut pas la confondre avec le modernisme qui se situe sur le plan de la

technologie alors que la modernité est une façon de concevoir les relations au monde. La modernité est le résultat đ'un long processus qui a vu, en particulier, la naissance de l'esprit scientifique avec la séparation sujet/objet, la coupure nature/culture,

l'apparition de la primauté de l'individu sur la société qui se manifeste dans le salut individuel du christianisme et débouche sur la Déclaration des droits de l'homme à la Révolution française. 
aux uns et aux autres les mêmes caractéristiques et en particulier la possession d'une âme, conception généralement désignée par le terme " animisme Mais le qualificatif " d'animiste " attribué à certaines sociétés résulte d'une vision réductrice qui s'est transformée peu à peu en un lieu commun.

Je me propose de montrer qu'il s'agit là d'une ignorance de la façon dont les sociétés non-modernes organisent les différents niveaux de la réalité. Nous verrons également comment ceux-ci correspondent à différents niveaux de fonctionnement du social.

Si l'on admet que l'on peut tirer des enseignements des savoirs populaires pour parvenir à une gestion raisonnée de la nature, il est nécessaire de comprendre comment ces savoirs se construisent et s'organisent. comment ils rendent compte de la façon dont la réalité est perçue, conçue et vécue dans chaque société. Ceci pose évidemment aussi la question de leur insertion dans le fonctionnement du social.

Les savoirs sont sous la double dépendance des capacités cognitives propres à l'espèce humaine et des caractéristiques du milieu dans lequel vit la société concernée ${ }^{3}$. Mais cette dépendance n'est pas absolue car les savoirs s'élaborent et se structurent dans le cadre des pratiques techniques et sociales. C'est ainsi que s'établissent les relations entre perception et conception, entre représentations et pratiques.

Mon objectif est ici de montrer que I'on ne peut aborder les modes de connaissance populaires sans les appréhender dans toute leur complexité ; pour cela, il faut tout d'abord distinguer les différents niveaux de réalité auxquels ils renvoient.

${ }^{2}$ C'est un argument utilisé. en particulier dans les critiques de la deep ecology ou des pourfendeurs de I'anthropocentrisme, critiques par ailleurs amplement justifiées.

${ }^{3}$ C'est ce que dit en particulier Lévi-Strauss (1982, p. 160).

${ }^{4}$ Notons qu'il peut $y$ avoir en outre dans certaines langues un classificateur indiquant la morphologie (arbre, herbe, liane, ...) et qu'il y a parfois ellipse du terme de base (reine des reinettes ou frisée à la place de pomme + reine

des reinettes et chicorée + frisée.

5 II faut remarquer que, dans un contexte urbain et sans aucune pratique de la nature de la part des personnes interrogées, les expériences menées par

E. Rosch ont montré que ce niveau de base se situe à celui du terme arbre, c'est-

à-dire ce qui ailleurs est une caractérisation d'une forme du vivant et non un terme d'appellation spécifique.
Un premier niveau est celui de la reconnaissance d'entités discrètes dans l'ensemble des êtres vivants et l'attribution d'un terme d'appellation. C'est ici qu'interviennent les processus cognitifs d'identification des objets dans leur diversité. Un deuxième niveau est celui de la façon dont chaque société établit un ordre dans cette diversité. Nous examinerons successive ment ces deux niveaux en envisageant principalement le monde végétal. Les exemples sont puisés dans différents types de sociétés, modernes et nonmodernes.

Nous aborderons enfin le problème de savoir à quel autre niveau du fonctionnement du social est apparue l'idée qu'il y aurait confusion, dans les sociétés non-modernes, entre les différents types d'êtres vivants, y compris les humains.

\section{La perception de la diversité des plantes et la question de la discontinuité dans le monde végétal}

\section{Découpage du réel, diversité et discontinuité}

La faculté de discrimination, puis de catégorisation, apparaît comme une caractéristique fondamentale des êtres vivants. Elle s'est perfectionnée avec le développement d'un système nerveux, puis d'un cerveau de plus en plus complexe et constitue un avantage adaptatif apparu au cours de l'évolution.

Chez l'homme, cette faculté de catégorisation est à la fois perceptuelle et conceptuelle et, de plus, elle s'inscrit dans la capacité qui lui est propre de communiquer avec ses semblables grâce au langage. Ainsi, la façon dont nous établissons des catégories dans le monde qui nous entoure, doit être envisagée comme un ajustement entre une aptitude innée et une compétence acquise au cours d'un apprentissage socialisé ; même si elle est influencée par les événements de notre histoire personnelle, il n'en demeure pas moins que les termes d'appellation que nous utilisons, par exemple pour désigner des plantes, doivent être compris par ceux à qui nous nous adressons.

C'est par l'attribution des termes d'appellation que l'anthropologue aborde la question de la reconnaissance de la diversité des plantes. Ces termes d'appelIation sont de deux types : a) termes de base seuls, simples comme hêtre, noisetier, frêne ou composés comme reine des prés ou bouton d'or ; b) termes de bases accompagnés d'un ou plusieurs déterminants. comme chêne + vert, épine + blanche, chou + de Milan ou carotte + rouge + longue + de Croissy ${ }^{4}$

La notion de terme de base est liée à celle de niveau de base qui, en dépit des nombreuses questions qu'il soulève, peut être considéré comme un niveau d'organisation stratégique sur le plan cognitif. Bien que ne pouvant pas être défini de façon absolue, il correspond à une réalité repérable par les psychologues dans des conditions expérimentales précises :

- Des expériences convergentes ont montré un niveau d'abstraction de base dans la classification humaine d'objets concrets. Les objets de base se sont avérés être les catégories les plus globales dont les membres se composaient de groupes d'attributs corrélés... Le nom du niveau de base était le nom le plus abstrait à partir duquel une image concrète peut être suscitée - (Rosch, 1976)).

Les quelques exemples donnés ci-dessus montrent que les distinctions les plus fines peuvent se situer soit au niveau de base quand la plante est désignée par un terme de base seul comme dans le cas du frêne, soit à un niveau taxinomique inférieur quand la plante est désignée par un terme de base accompagné d'un ou plusieurs déterminants ; c'est le cas pour chêne + vert ou carotte + rouge + longue + de Croissy $^{5}$

Ce fait a entraîné deux types de débats en ethnoscience : l'un sur le statut de ce qu'il faut considérer comme unité perceptuelle élémentaire ; l'autre sur la question des limites entre les taxons. Ces limites rendent-elles compte de la perception d'une réelle discontinuité entre les entités ou s'agit-il d'un découpage arbitraire dans un continuum ?

Nous abordons là un problème dont on débat depuis longtemps. Lévi-Strauss disait dans La Pensée sauvage :

- La diversité des espèces fournit à l'homme l'image la plus intuitive dont il dispose et elle constitue la manifestation la plus directe qu'il sache percevoir de la discontinuité ultime du réel - (Lévi-Strauss, 1962, 181). 
Cependant, avant même la mise en évidence des principes de l'Évolution et avant la découverte du support génétique des caractères, Buffon disait

-...Pour faire un arrangement, un système, en un mot une méthode générale, il faut que tout $\mathrm{y}$ soit compris ; il faut diviser ce tout en différentes classes, partager ces classes en genres, sous-diviser ces genres en espèces et tout cela suivant un ordre dans lequel il entre nécessairement de l'arbitraire. Mais la nature marche par des gradations inconnues et par conséquent elle ne peut pas se prêter totalement à ces divisions, puisqu'elle passe d'une espèce à une autre par des nuances imperceptibles... - (1752 : 17-18).

Tout ceci tend à prouver que la façon dont on conçoit le monde comme continu ou discontinu est autant ou sinon plus un problème conceptuel que perceptuel, ainsi que nous le montre l'histoire des sciences en Occident ${ }^{6}$.

Pour ce qui est de la perception des êtres vivants, on peut estimer que l'idée d'un continuum, avancée par Buffon, a été corroborée par la théorie darwinienne de l'évolution, mais on peut aussi affirmer que la découverte des supports génétiques indique que, même si on peut percevoir les apparences des phénotypes comme " une gradation - de * nuances imperceptibles ", il y a une discontinuité au niveau des gènes.

De toute façon, le continuum des apparences ne peut s'imposer à l'observateur que dans un certain nombre de cas : les organismes intermédiaires n'existent simultanément en un même lieu que durant un temps limité et la chaîne des êtres, résultat de l'évolution, est une reconstruction à partir des découvertes des paléontologues. Quant aux intermédiaires dus à des hybridations, ils ne sont possibles dans la nature qu'à l'intérieur d'une même espèce et quelquefois seulement, entre certaines d'entre elles ${ }^{7}$.

C'est-à-dire qu'il est difficile d'arguer de la difficulté qu'ont parfois les biologistes, qui traitent de l'ensemble des êtres de la planète, à définir la notion d'espèce et à délimiter certaines d'entre elles, pour en tirer la conclusion qu'une population donnée, face à un environnement naturel donné, effectue dans un continuum un découpage arbitraire qui peut ne pas être le même pour tous ${ }^{8}$.

En revanche, on peut s'interroger, dans un contexte écologique et social particulier, sur la façon dont se comportent les populations face à leur environnement végétal. Quels sont les types de plantes qui constituent des entités indiscutables sur lesquelles tous s'entendent pour les appeler du même nom et à quel niveau taxinomique les situent-ils ? Quels sont ceux sur lesquels il y a hésitation et avis contradictoires ?

\section{Critères de reconnaissance d'entités discrètes et modes d'appellation pour les plantes sauvages.}

Pour mieux caractériser les différents types de cas que l'on peut rencontrer, nous nous référons à la nomen- clature scientifique qui établit un regroupement en genres à partir d'espèces distinctes à l'intérieur desquelles on peut reconnaître des unités taxinomiques de rang inférieur, par exemple des sousespèces ou des variétés. Je m'efforcerai de donner quelques exemples en français afin de fixer les idées ${ }^{9}$, les autres seront principalement empruntés à la nomenclature bunaq ${ }^{10}$.

Quelle que soit la société sur laquelle on travaille, on trouve toujours un certain nombre de plantes auxquelles toutes les personnes interrogées attribuent, sans hésitation, le même terme d'appellation. C'est par exemple, en île de France, le cas d'un certain nombre d'arbres communs : hêtre, noisetier, châtaignier, frêne ou d'une plante lianescente comme le tamier (Tamus communis). Il s'agit là de genres botaniques monospécifiques pour la région, dont le patrimoine génétique est suffisamment éloigné de toutes les autres plantes pour que leur apparence phénotypique soit totalement différente. Mais il faut remarquer que tous les genres monospécifiques ne sont pas forcément repérés. Par exemple une Orchidée de sous-bois comme Lystera ovata qui est d'un aspect remarquable pour le botaniste, n'est ni reconnue, ni nommée par les paysans du Gâtinais.

Dans le cas de genres polyspécifiques dans une région donnée, on relève plusieurs possibilités pour nommer les espèces d'un même genre :

a) elles portent le même terme de base avec des déterminants différents pour chaque espèce, ce qui correspond au binôme * genre + espèce - de la nomenclature scientifique ${ }^{11}$. C'est, par exemple, le cas pour les pins dans le Gâtinais : Pinus sylvestris est appelé sapin + rouge et le Pinus nigra (le pin d'Autriche qui est une espèce introduite), sapin + gris.

b) des termes de base différents leur sont attribués : ainsi (toujours dans le Gâtinais) cornouiller pour le Cornus mas et sanguin pour le Cornus sanguinea.

c) elles sont désignées par un seul terme de base sans déterminant : c'est, en France, le cas pour toutes les espèces sauvages de Rubus appelées ronces (là où il n'y a pas de framboisiers sauvages).

d) plusieurs espèces ont pour appellation un même terme de base sans déterminant tandis qu'une autre espèce remarquée pour une raison ou une autre est désignée par un autre terme de base. Ainsi la plupart des Viola, de couleur uniforme, sont appelées violettes, alors que la Viola tricolor est dénommée pensée + sauvage ; l'aspect joue sans doute ici un rôle, mais aussi le fait que les premières se trouvent dans ou aux abords des bois et la dernière dans les champs. De même Salix viminalis qui sert en vannerie est distingué des autres saules en étant désigné par le terme de base osier.

En revanche certains termes de base regroupent des espèces appartenant à des genres et même des familles différentes. Par exemple, Eryngium campestre (figure 1) qui est une Apiacée est désignée, en raison de ses piquants, par le même terme de base chardon que d'autres plantes herbacées également piquantes, mais appartenant à une famille botanique totalement différente, celle des Astéracées (figure 2). Ces plantes sont souvent distinguées, mais pas forcément, par des déterminants différents ; ainsi l'Eryngium est
${ }^{6}$ En effet, depuis I'antiquité grecque, on a opposé une " réalité vraie "que nous pourrions atteindre grâce à notre raison et une apparence qui serait le produit de notre perception sensible, comme si la raison pouvait se forger une idée du monde qui soit indépendante des données que nous fournissent nos sens. Ce débat s'est trouvê ravivé au début de ce siècle quand les physiciens ont découvert la preuve de l'existence de particules élémentaires, c'est-à-dire de la discontinuité de la matière et, en même temps, que la question de l'existence de cette particule en tant qu'entité n'avait pas réellement de signification

(E. Schrödinger, 1992). On s'est alors à nouveau interrogé sur les rapports entre ce que l'esprit humain perçoit et ce quill conçoit et sur la façon dont la théorie atomiste est apparue dans la pensée grecque dès le ve siècle avant notre ère chez Leucippe et Démocrite. II ne s'agissait pas là évidemment du résultat d'une observation directe, mais d'un raisonnement logique à partir d'une constatation sur la manière dont la matière occupe le vide d'une façon plus ou moins dense.

7 Toutefois beaucoup plus fréquemment dans le règne végétal que dans le règne animal. 
appelé chardon + Roland, tandis que les diverses espèces d'Astéracées (Carduus, Cirsium, Onopordon, ...) sont souvent simplement appelées chardon. Autre exemple, celui du terme ortie appliqué à des Urticacées, mais aussi à une Labiée dont les feuilles non urticantes ont le même aspect ; cette dernière est appelée ortie + blanche en raison de la couleur de ses fleurs et par opposition aux Urticacées qui sont apétales.

Si nous examinons maintenant quelques exemples de la nomenclature bunaq, nous y trouvons des similitudes avec ce que nous venons de voir pour la France, c'est-à-dire l'attribution d'un même terme de base pour des plantes ayant des caractéristiques communes. Celles-ci peuvent correspondre à des affinités reconnues également par la classification scientifique comme nous l'avons vu plus haut pour les Pinus du Gâtinais, mais pas forcément.

Ainsi, les deux espèces du genre Alstonia portent le même terme de base zoil, zoil + belis " blanc " pour A. scholaris, et zoil + guzu * noir * pour A. spectabilis. En revanche, sur les cinq Cassia existant sur le territoire bunaq, seuls deux qui sont des arbres proches par le port, les feuilles et les fleurs, portent le même terme de base avec des déterminants différents arus + mone " masculin "pour Cassia javanica, arus + pana - féminin - pour $C$. fistula. Les trois autres, dont

${ }^{8}$ Comme le dit par exemple R. Ellen (1993), it est vrai à propos de la classification des animaux. l'aspect est différent (deux sont des arbustes de petite taille), portent chacun un autre terme de base.

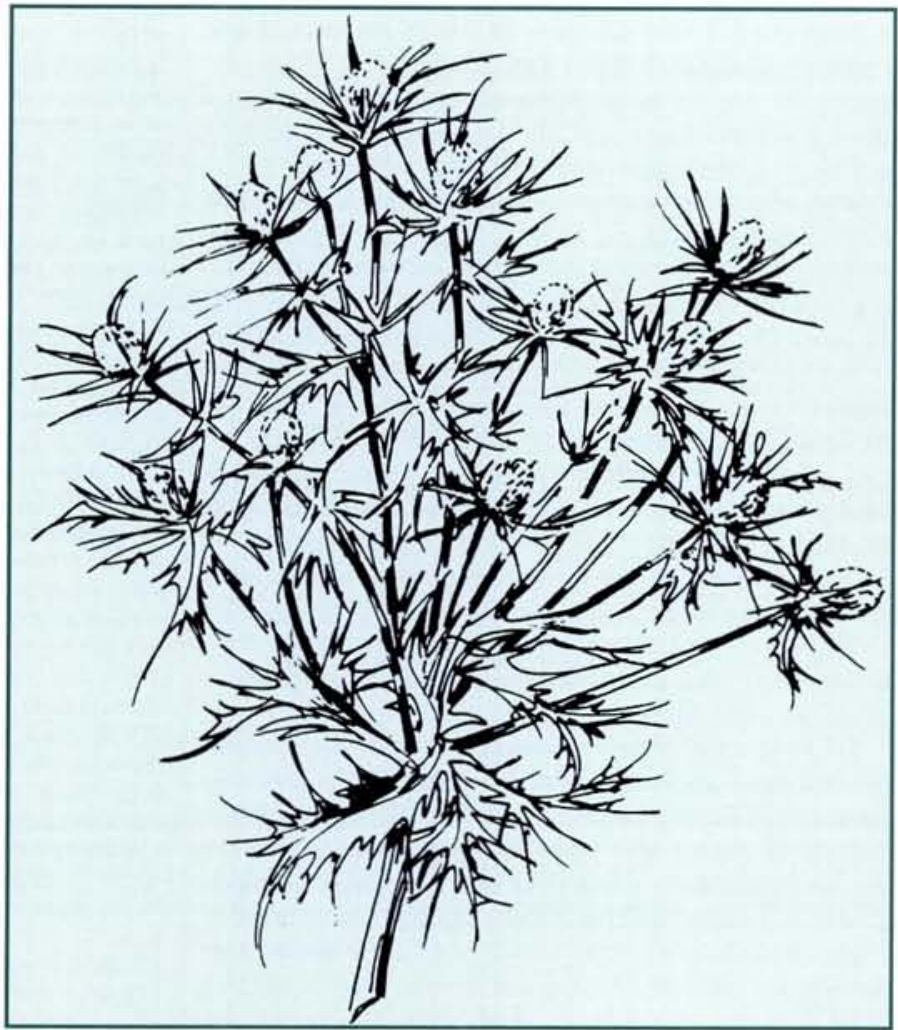

Figure 1. Eryngium campestre (Apiacées).
Cependant, l'attribution d'un même terme de base peut se fonder sur une ressemblance morphologique qui peut se borner à un caractère seulement. Par exemple l'appellation e, qui désigne en premier lieu un arbre Albizia chinensis, est aussi donnée à plusieurs plantes possédant le même type de feuilles pennées, mais qui sont des herbacées : e + gol * + petit " Aeschynomene indica et e + gol + lotu * + petit + court " Smithia sensitiva ; toutes appartiennent à la même famille botanique, celle des Fabacées. Mais le même terme de base peut aussi être donné à des plantes appartenant à des familles différentes. Par exemple le terme ukaq est utilisé pour deux Asclépiadacées, ukaq + masaq * + grand * Calotropis gigantea et ukaq + lotu * + court " Asclepias curassavica ; mais, accompagné du déterminant gol * petit il est également attribué à des petites Euphorbiacées : Euphorbia prostata et Phyllantus ninuri. Le seul point commun à toutes ces plantes est d'avoir du latex et de ne pas être des arbres.

Le cas des Ficus est particulièrement intéressant : il y a, d'une part, tous les - étrangleurs "qui commencent leur existence comme épiphytes ; ils sont désignés par le terme de base pur avec chacun un déterminant différent. II y a ensuite tous les autres Ficus arborés qui portent chacun un terme de base différent, certain sans déterminant kabokeq, tau, teor, sobor, tandis que d'autres sont regroupés sous le terme erol. Pourtant, il y a hésitation quand on ne connaît que la forme adulte et que l'on ne sait pas si l'arbre a commencé son existence comme épiphyte : c'est le cas des $F$. tinctoria ssp. gibbosa var. rigida - étrangleurs * que j'ai rencontrés à 1000 m d'altitude autour des places réservées aux réunions rituelles oủ il reste petit, alors que, plus bas, cette espèce peut atteindre $45 \mathrm{~m}$ de haut ; je l'ai récolté avec plusieurs appellations : soit pur + sait * (qui) gratte " ou erol + sait sans doute par a contamination - avec erol + guzu - noir *, $F$. ampelas qui a aussi des feuilles râpeuses et avec lequel on le confond également. Cependant, les Bunaq reconnaissent à tous ces Ficus des caractéristiques communes : le même type de fruits et le même type de bourgeon terminal et de disposition des feuilles.

\section{Aspect fonctionnel des distinctions et des rapprochements}

Nous voyons ainsi se dessiner à travers la dénomination populaire des plantes un certain nombre de principes dans la façon dont sont établis des rapprochements et des distinctions entre les diverses espèces végétales dans un environnement donné. Un rapprochement peut conduire à donner le même terme de base à des plantes que l'on distingue cependant par des déterminants différents ; ce qui n'est pas la même chose que de regrouper de façon indifférenciée des plantes ayant quelque chose en commun et pour lesquelles on juge soit qu'on ne peut établir de distinction, soit, si on les différencie, qu'il n'est pas nécessaire 
de donner des déterminants. C'est généralement le cas de types de plantes qui ne jouent aucun rôle particulier individuel et que l'on peut traiter de façon collective ; c'est le cas signalé plus haut des violettes en France ou celui de différentes espèces de Strobilantes de sous-bois, toutes appelées matenebuq par les Bunaq.

Les rapprochements et les distinctions sont souvent fondés sur l'aspect morphologique, mais aussi sur d'autres caractéristiques biologiques de la plante : anatomique, physiologique, écologique. Un rapprochement peut correspondre à une proximité sur le plan botanique, mais c'est loin d'être toujours le cas. II peut s'agir de similitude dans l'aspect (c'est le cas pour ortie et chardon) ou bien d'un même comportement ; par exemple plusieurs mauvaises herbes appartenant à des genres différents de la famille des Poacées ayant toutes la particularité d'avoir des rhizomes difficiles à arracher sont dénommées chiendents. Fondé sur des critères limités, le regroupement peut aussi s'effectuer autour d'une plante ayant une importance culturelle : c'est le cas pour ukaq, chez les Bunaq, oủ l'espèce éponyme, Calotropis gigantea, est une espèce pionnière, qui à ce titre, figure dans les mythes parmi les plantes qui sont apparues en premier sur terre. C'est aussi le cas en français de plusieurs arbustes ayant des feuilles persistantes approximativement de même forme qui sont appelés laurier, du nom du prestigieux Laurus nobilis avec des déterminants différents (rose, tin, ...).

Pour ce qui est de l'usage, il peut justifier que l'on donne à la plante un terme de base particulier comme nous l'avons vu en français pour l'osier par opposition aux autres saules. Au contraire, comme c'est souvent le cas pour des plantes introduites, l'usage peut entraîner l'attribution d'un terme de base existant déjà dans la langue. Ainsi, dans la nomenclature bunaq, le piment, en raison de son utilisation comme condiment piquant, s'est vu attribuer lors de son introduction, le même terme de base (patal) qu'un Piper sauvage, $P$. retrofactum. C'est d'ailleurs aussi le cas dans d'autres régions d'Indonésie, à Java et Bali (cabe et tabia).

Remarquons que certaines distinctions qui ne sont pas prises en compte par les botanistes le sont par les Bunaq. Ainsi pour Pittosporum moluccanum, là oủ les botanistes ne voient qu'une seule espèce polymorphe en fonction des conditions écologiques, les Bunaq voient deux types d'arbre portant le même terme de base ziek, l'un à feuilles sombres ziek + guzu * + noir - l'autre à feuilles claires ziek + belis * + blanc *

Pour mieux saisir la façon dont les populations locales établissent des distinctions entre types de plantes, on peut également s'interroger sur leur attitude vis-à-vis des hybrides qui se forment spontanément dans la nature.

Les Bunaq ne m'ont jamais signalé le cas de types intermédiaires entre pie, Eucalyptus alba, et tal, E. urophylla, espèces qui présentent de nombreux cas d'hybridation à Timor (Martin et Cossalter 1975-76), mais que je n'ai jamais rencontrées à Lamaknen.

Pour ce qui est de la France, examinons le cas des chênes. II en existe trois espèces qui se ressemblent beaucoup : Quercus pubescens, $Q$. petraea, et $Q$. robur ;

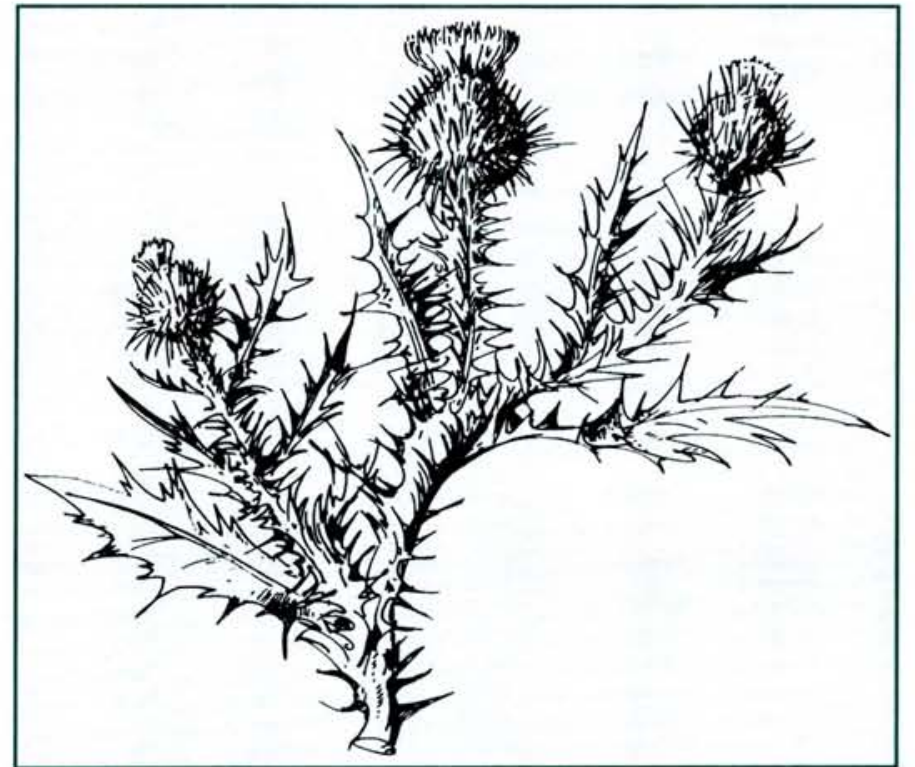

Figure 2. Astéracées : Onopordon acanthium.

en outre, les botanistes disent que ces trois espèces s'hybrident. En dehors de la période de floraison et de fructification, il leur est impossible de dire à quelle espèce appartient un arbre, car la différence entre les deux dernières espèces est une question d'absence ou de présence de pédoncule dans la fleur. Dans le Gâtinais, alors que les botanistes ont des difficultés à identifier les espèces récoltées, les informateurs, eux, distinguent deux types de chêne, le grisard et le blanc ; ce dernier se caractériserait par un tronc plus lisse, différence très difficile à apprécier quand on entre pour la première fois dans un bois ; on peut se demander à quelle espèce ou à quel hybride correspondent ces termes et douter qu'il existe des critères morphologiques végétatifs qui permettent de les distinguer. La véritable distinction se situe sur le plan du comportement et de la qualité du bois : le blanc pousse plus vite et brûle aussi plus rapidement. II s'agit donc là d'un savoir lié à la connaissance de la régénération et de la dynamique de formations arborées, exploitées sous forme de taillis essentiellement pour le bois de chauffe.

Le repérage d'une discontinuité apparaît donc ici comme une nécessité pratique. Cette volonté de distinction peut aller jusqu'à donner des termes de base différents pour des formes appartenant à la même espèce. Ainsi, les Haoussa distinguent, selon leurs caractéristiques écologiques, deux formes d'Acacia nilotica qui portent chacune un terme de base différent : marji pour $A$. nilotica nilotica qui affectionne les zones inondées et bagaruwa pour $A$. nilotica adansoni qui ne les supporte pas (Luxereau et Roussel, 1995).

D'une façon générale, on peut dire que l'accent est mis sur la discontinuité chaque fois qu'il est nécessaire de distinguer un type de plante jouant un rôle particulier dans les usages techniques ou culturels, ou
9 Les exemples donnés en français sont de deux types: a) il s'agit de termes d'appellation en français courant que l'on peut trouver dans les dictionnaires. Ils sont destinés à faciliter la compréhension des lecteurs mais ne peuvent en aucun cas être considérés comme appartenant à un système d'appellation propre à une société particulière. En effet, ces termes correspondent à des appellations originaires de plusieurs régions de France et qui ne sont pas forcément utilisés par les mêmes personnes ; b) des termes recueillis lors de deux recherches de terrain effectuées dans le cadre du comité - Systèmes ruraux * du Pir - Environnement, vie et société - du CNRS: Usages des sols, pratiques d'éleveurs, représentations de la nature et dynamique des milieux et des ressources (responsables : Marianne Cohen et Sylvie Lardon) et Devenir des ilots boisés dans les plaines de grande culture: l'exemple du Gâtinais occidental (responsable : Patrick Blandin). Leur origine sera signalée. Pour des renseignements plus complets sur ces recherches, on pourra consulter les rapports signalés dans la bibliographie. 
comme repère sur le plan écologique. II y a indifférence à cette même discontinuité quand celle-ci n'a pas de fonction spécifique, sur un plan ou sur un autre, dans la vie des membres de la société concernée.

Ainsi, face aux différentes herbacées qui composent un pâturage naturel et dont ils admettent la diversité mais qu'ils traitent de façon collective, les éleveurs du Causse Méjan ne vont distinguer par des termes d'appellation particuliers que celles qui sont très appréciées ou au contraire complètement refusées par les moutons, ou encore celles qui sont comestibles pour I'homme. Là aussi, on peut noter des termes d'appellation différents pour des espèces d'un même groupe (Festuca ovina) : Festuca duriuscula spp. duriuscula que les brebis n'apprécient pas est appelée la bourre, tandis que Festuca duriuscula ssp. glauca est appelée I'herbe douce (Friedberg, 1996, p 35).

En revanche, face à une diversité dont l'évidence semble s'imposer sur le plan morphologique, il peut y avoir un regroupement, fondé sur quelques caractéristiques communes, autour d'un terme de base éponyme, là aussi pour des raisons fonctionnelles mais, cette fois-ci, sur le plan cognitif, l'objectif étant un effort d'économie de mémoire. C'est ce que nous avons vu, chez les Bunaq, pour ukaq ou, en français, pour laurier.

Ce phénomène s'observe couramment pour les plantes cultivées introduites qui sont généralement désignées par le terme de base d'une plante autochtone avec laquelle on peut établir une vague ressemblance sur le plan morphologique ou faire état d'un usage comparable. Nous avons vu le cas du piment chez les Bunaq ; signalons en français, l'attribution du terme pomme à différentes plantes comestibles introduites d'Amérique : pomme de terre, pomme d'amour, pomme cannelle...

D'une façon générale, on peut constater l'application d'une règle s'apparentant curieusement au principe de parcimonie dans les classifications scientifiques établissant entre les êtres des relations les plus simples possible. Dans la nomenclature populaire, il s'agit d'un souci de rapidité dans le repérage et l'identification et d'économie dans l'effort de mémorisation.

Une distinction peut ou non s'imposer sur le plan perceptuel ; pour être retenue, il faut qu'elle ait un sens sur le plan conceptuel, ce qui implique une prise en compte du rôle fonctionnel de la plante.

Les découvertes faites dans le cadre des neurosciences nous apprennent qu'une partie au moins des processus cognitifs en jeu dans la reconnaissance des objets et des visages, se déroulent en dehors de la conscience. Ceci doit nous inciter à une grande prudence dans l'établissement de liens entre ces processus cognitifs et la façon dont s'organisent les savoirs. Ceux-ci ont pour fonction primordiale de s'inscrire dans des pratiques et ils doivent, de façon tout aussi impérative, permettre de communiquer avec autrui.

Ainsi, dans le Gâtinais, l'absence d'accord entre les personnes interrogées sur les termes d'appellation de nombreuses espèces forestières est sans doute un des effets de l'effondrement de la société rurale. Les quelques individus qui continuent à fréquenter les bois pour les exploiter ont un savoir correspondant à des pratiques techniques individuelles qu'il n'est plus nécessaire de partager avec d'autres.

\section{Diversité et discontinuité : le cas des plantes alimentaires cultivées}

Après avoir examiné le cas de la diversité des plantes sauvages sur laquelle les hommes ne peuvent agir directement, voyons maintenant ce qu'il en est, pour les plantes cultivées, sur la diversité et la discontinuité desquelles chaque acteur peut intervenir. Nous nous limiterons ici au cas des plantes alimentaires.

Souvent, pour ces plantes, les termes de base correspondent à l'espèce et le terme de base + un déterminant à des variétés ; c'est-à-dire que la diversité se situe ici au niveau infraspécifique. C'est, par exemple, le cas de la carotte, Daucus carota, ou celui du chou, Brassica oleracea ; ainsi pour ce dernier, nous avons le chou + fleur ( $B$. oleracea var. botrytis), chou + de Milan ( $B$. olerecea var. capitata), chou + de Bruxelles ( $B$. oleracea var. gemmifera).

Pour les plantes cultivées, il existe deux types d'attitude

a) ou bien on met au point, par sélection, des variétés ayant chacune des caractéristiques particulières que l'on s'efforce de conserver d'une génération sur l'autre. Jusqu'à ce que la science donne accès à la connaissance des mécanismes génétiques, il s'agissait évidemment de pratiques empiriques fondées sur les phénotypes :

b) ou bien, au contraire, on cultive des * populations ", c'est-à-dire des ensembles d'individus ayant des formules génétiques différentes et qui s'hybrident entre eux à chaque génération. Dans ce cas, l'objectif est de cultiver des ensembles présentant une certaine souplesse face aux aléas climatiques. C'est principalement pour les céréales semées et récoltées de façon globale que l'on cultivait des populations ; c'était jadis le cas pour le blé en France ; mais c'est aussi ainsi que les Bunaq cultivent le riz, dont ils reconnaissent pourtant plusieurs variétés, mais qu'ils cultivent ensemble dans le même champ ; ils disent euxmêmes qu'il y a hybridation en reconnaissant qu'une variété récemment introduite s'est transformée au contact de celles qu'ils cultivaient jadis ; extérieurement, elle a toujours le même aspect, mais intérieurement la couleur du grain a changé.

II arrive également que pour des légumes que l'on reproduit par graines, on ne se soucie pas d'avoir des variétés bien délimitées, mais c'est plutôt la première alternative qui prévaut et c'est évidemment aussi le cas pour toutes les plantes que l'on reproduit végétativement.

L'avantage de cultiver des variétés bien distinctes est de pouvoir repérer exactement les conditions de milieu qui leur sont favorables et de connaître les qualités organoleptiques et culinaires des parties utilisées. 
Les variétés locales correspondent souvent à certaines préparations culinaires liées à l'idée qu'un groupe social se fait de son identité culturelle. C'est-àdire que dans ce cas, non seulement il y a reconnaissance d'une diversité, mais en plus effort pour maintenir une discontinuité en sélectionnant les semences et en pratiquant des cultures séparées pour empêcher les hybridations. Évidemment le maintien de la discontinuité est plus facile à obtenir dans le cas d'une reproduction végétative qui permet d'obtenir des plantes identiques d'une génération à l'autre. Cette attention à maintenir la discontinuité est particulièrement indispensable quand certaines variétés sont nécessaires à l'exécution des pratiques rituelles.

Ainsi, contrairement aux Bunaq, les Balinais prennent soin de semer et récolter séparément les variétés de riz dont ils ont besoin pour exécuter les rituels et en particulier chaque fois qu'il faut mettre du riz de couleur correspondant aux quatre Orients : noir au nord, rouge au sud, blanc à l'est et jaune à l'ouest (dans ce cas il s'agit de riz blanc coloré au Curcuma (figure 3)). Mais sont également nécessaires quatre variétés de noix de coco (figure 4) et quatre de banane correspondant chacune à une des quatre couleurs auxquelles il faut ajouter, pour le centre, une variété censée les posséder toutes les quatre.

Lexigence d'une variété particulière de plante cultivee pour accomplir un rituel se retrouve dans de nombreuses populations, ce qui pose évidemment des problèmes quand les services agronomiques locaux fournissent des variétés qui ont été sélectionnées à l'extérieur.

\section{L'ordre de la nature}

Nous abordons ici un autre niveau de classification qui consiste à mettre de l'ordre dans l'ensemble des plantes portant des termes de base différents.

Deux types d'attitudes sont possibles qui généralement coexistent et peuvent même influer l'une sur l'autre

a) ou bien cette mise en ordre consiste à faire entrer les différents êtres vivants ou une partie de ceux-ci dans des catégories construites sur des critères choisis a priori. Il s'agit alors d'organiser l'ensemble des éléments de l'univers en établissant des corrélations de type symbolique ou métaphorique entre des éléments appartenant à des domaines différents

b) ou bien il s'agit d'organiser les éléments du monde végétal à partir de critères déduits de l'observation et liés aux pratiques dont chaque type de plante est l'objet.

Dans le premier cas, il s'agit de classifications regroupant des objets de nature différente, mais possédant aux yeux des membres de la société concernée des caractéristiques permettant d'établir entre eux des correspondances.

Ces classifications jouent un rôle non seulement dans les relations que l'on entretient avec les plantes, mais également dans le fonctionnement global de la société. Ce sont ces types de classification qui ont tout d'abord frappé les ethnologues. Ce sont elles

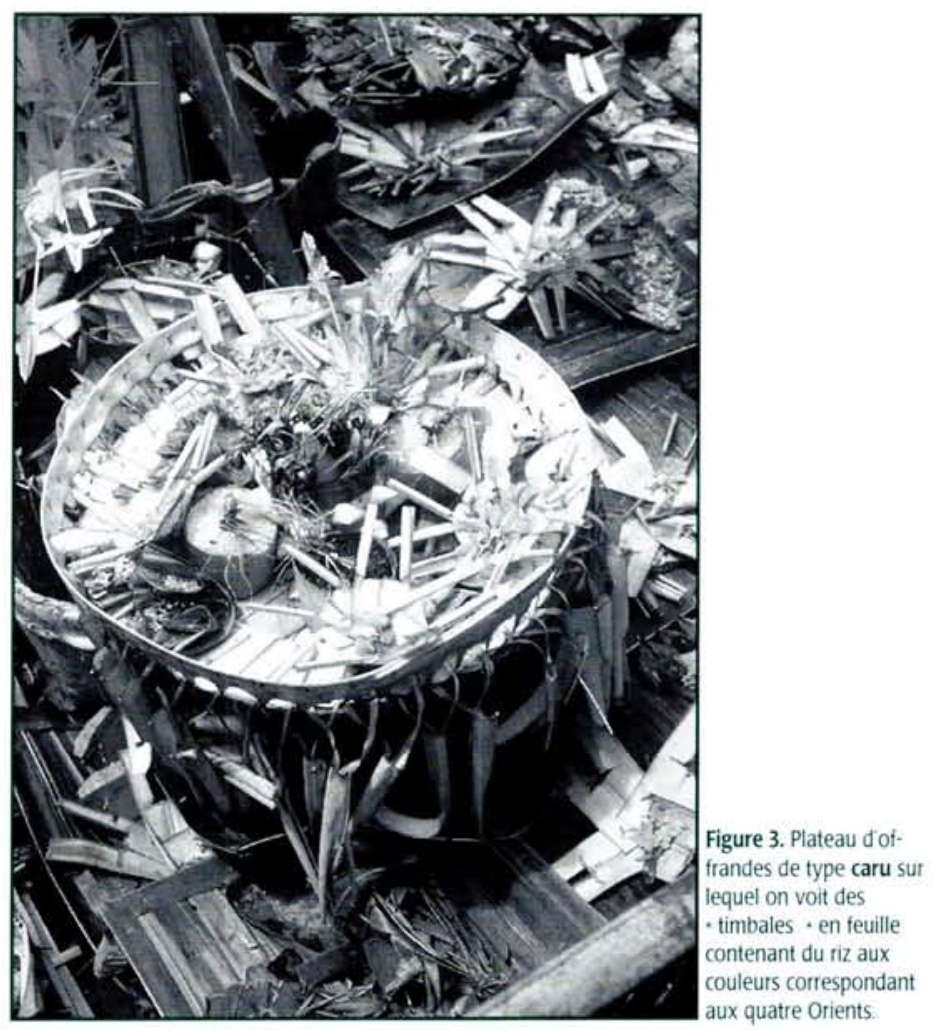

NATURES SCIENCES SOCIETES 1997

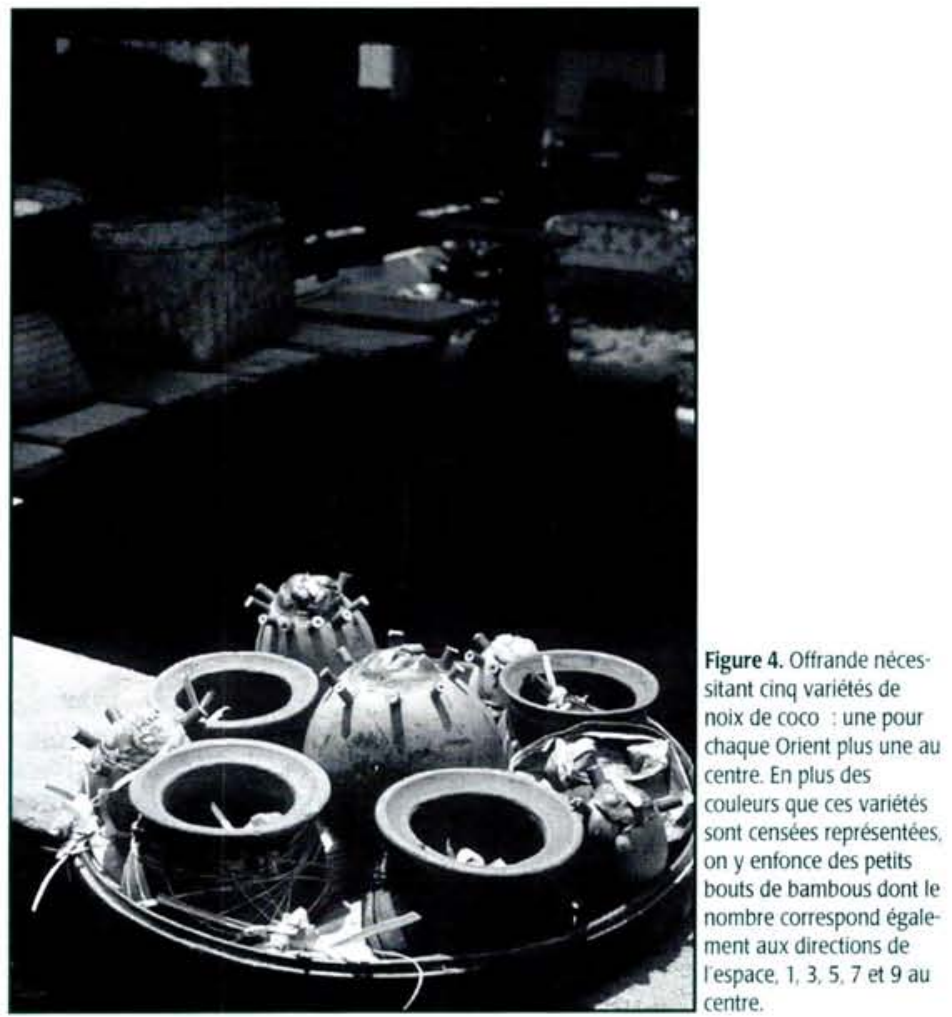


dont il est question dans l'article de Durkheim et Mauss (1901) qui traite pour la première fois de ces questions et fournit des exemples pris chez les Indiens d'Amérique du nord et les Aborigènes australiens. On y voit, par exemple, mis dans une même catégorie, des " clans ", des "fratries ", des animaux, des plantes et une direction de l'espace.

Comme nous l'avons vu plus haut, c'est en relation avec ce type de classification que les Balinais sont attentifs à la préservation de leurs variétés de riz, de bananiers ou de cocotiers nécessaires à certaines offrandes, en particulier celles destinées à figurer périodiquement une remise en ordre des choses, des êtres et des divinités en fonction des différents orients. II faut ajouter que pour d'autres offrandes, ce sont des plantes ou des animaux appartenant à des catégories fondées sur le milieu qu'il est nécessaire de faire figurer comme représentation de la diversité du vivant.

Dans le second cas, la classification porte uniquement sur les plantes, c'est à dire sur des objets que les acteurs considèrent comme étant de même nature. Cependant, dans les classifications populaires qui nous intéressent ici, nous n'avons pas affaire à une volonté délibérée et explicite de classer, comme c'est le cas pour les classifications savantes et en particulier pour les classifications scientifiques. Ce sont les chercheurs qui, grâce à leurs analyses du vocabulaire et leurs enquêtes auprès des acteurs, mettent en évidence l'existence de catégories. D'une façon générale, ces dernières ont une fonction opératoire aussi bien sur le plan cognitif que pratique.

Certaines de ces catégories sont fondées sur des critères morphologiques, biologiques ou écologiques, permettant de repérer les plantes dans la nature. II en va ainsi chez les Bunaq avec * les plantes à wo ", (ce sont des plantes sans tige comme les Aracées dont les feuilles, réunies à la base, forment ce que les Bunaq appellent des wo) ; ou lepu guk * entrenœuds nœuds ", ensemble comprenant toutes les Poacées, les Palmiers et quelques Astéracées et Acanthacées à feuilles opposées ; ou encore hotel il bul * arbre eau base ", c'est-à-dire des arbres qui peuvent attirer l'eau et la mettre en réserve, ce qui leur permet de pousser dans des endroits secs. Cet ensemble comprend les Ficus qui appartiennent aussi à l'ensemble hotel susuqil - arbre à latex ". En effet dans les classifications populaires, les plantes peuvent appartenir à plusieurs catégories à la fois (figure 5).

Mais il y a aussi des catégories pour lesquelles les caractéristiques intrinsèques de la plante sont associées à des critères liés aux relations que les membres de la société entretiennent avec elle. Ainsi on trouve chez les Bunaq la catégorie hotel deu goq * arbre maison produit = contenant des arbres utilisés dans la construction, caractérisée par un morphotype * arbre - et un usage, ou celle des plantes à tubercules comestibles appelée balo dik " taro, igname ". Quand d'autres critères, appartenant chacun à des domaines différents, $s^{\prime} y$ ajoutent, on peut alors parler de catégories complexes.

Par exemple, toujours chez les Bunaq : hotel upan gutu * arbre avec un pétiole (upan) comme celui des palmiers ou des bananiers ", qui rassemble donc des végétaux caractérisés par une certaine caractéristique morphologique, mais également par le fait que ce sont des plantes á usages multiples placées sous la protection des " gardiens des produits du sol * ; la catégorie bula est un autre exemple : elle est définie par un certain nombre de types de plantes caractéristiques de la prairie, mais le terme bula désigne aussi l'ensemble des terres oủ le bétail peut se déplacer en toute liberté, comprenant aussi des espaces arborés, et s'oppose ainsi aux terres réservées aux cultures qui lui sont interdites ; c'est-à-dire que bula correspond à un certain statut sur le plan foncier.

En France, on trouve aussi ce type de catégorie complexe. II en va ainsi, par exemple, de la catégorie lande qui peut être définie par une certaine composition floristique, mais qui correspond aussi à un certain type d'usage et peut également avoir un statut foncier particulier. C'est le cas également du terme brousse qui désigne un type de végétation formée de ligneux bas, souvent épineux, associés à des herbacées et dont la caratéristique est de se développer là oủ les hommes ne contrôlent plus la végétation : talus de chemins ou espaces agricoles qui ne sont plus entretenus. Autre exemple, dans le causse Méjan, le terme couderc désigne de bons prés entretenus par les brebis qui y sont menées de façon régulière ; elles s'y attardent parce que l'herbe y est bonne et favorisent ainsi la repousse ; en outre, elles y laissent leurs excréments ce qui entretient la fertilité. Ces coudercs se trouvent sur des bas de versants où se sont accumulés des sédiments argilolimoneux ; le sol y est profond et n'est donc pas fragilisé par le passage fréquent des brebis. Le couderc est donc le produit d'une combinaison complexe entre un sol, une végétation, une pratique pastorale et une certaine organisation de l'espace socialisé (op. cit. p 10).

Ainsi les catégories complexes sont souvent des catégories organisatrices de l'espace, qui prennent une importance prépondérante dans les milieux anthropisés, oủ ils sont associés à des pratiques ; mais elles jouent également un rôle sur le plan cognitif en constituant des ensembles à l'intérieur desquels on peut établir des distinctions entre des plantes portant des termes de base différents.

II faut noter que dans les milieux très anthropisés, les toponymes prennent souvent le relais des catégories complexes dans l'organisation de l'espace.

\section{Unité et continuité du vivant}

Comme nous l'avons annoncé dans l'introduction, nous ne traitons plus ici seulement des plantes, mais de l'ensemble des êtres vivants, y compris l'homme. Le concept d'unité et de continuité du vivant que l'on trouve dans la plupart, sinon dans toutes les sociétés non-modernes, n'est pas contradictoire avec ce que nous venons de dire sur la reconnaissance de la diversité et de la discontinuité puisqu'il se situe à un autre niveau englobant le fonctionnement de l'ensemble de la société considérée, c'est-à-dire hiérarchiquement supérieur. À ce niveau, il ne s'agit plus des 


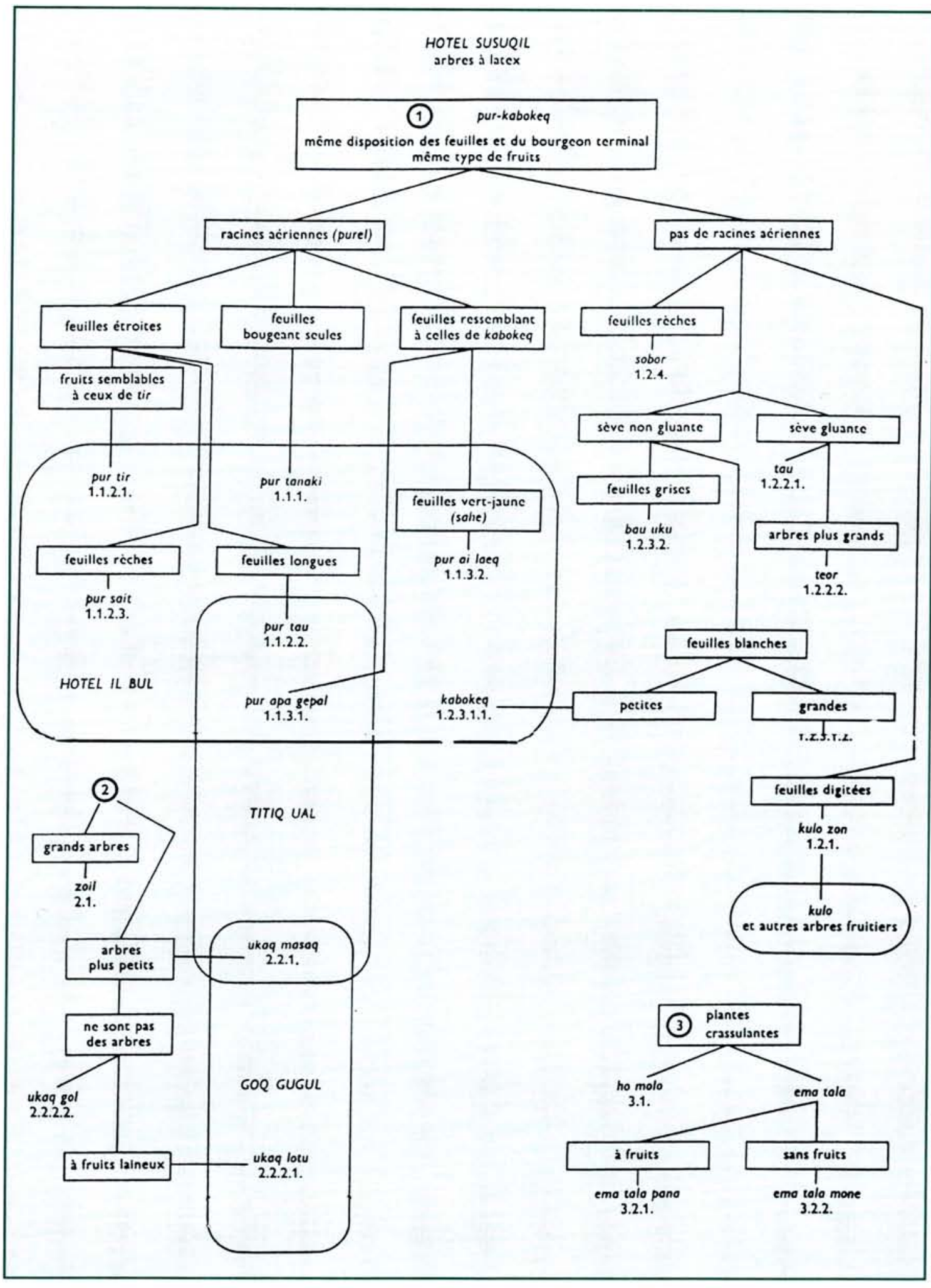


individus constituant la diversité, mais de la façon dont chaque société conceptualise les éléments qui composent ces individus, conditionnent leur naissance puis leur devenir au cours de leur existence et au moment de leur mort.

Plusieurs catégories d'êtres peuvent être reconnues en fonction :

a) des différences dans leurs composantes,

b) de la manière dont ces dernières se transforment au cours de la vie et après la mort,

c) de la position que ces êtres occupent dans le réseau des relations qui fondent leur identité dans le cadre général du fonctionnement de la société.

Parmi les composantes des êtres vivants les plus connues sont les âmes. Dans certaines sociétés oủ la chasse joue un rôle important, ces dernières rejoignent, à la mort des individus, un stock fixe et indifférencié et vont à nouveau se réincarner dans des individus qui peuvent être humains ou animaux ${ }^{12}$. Mais il existe bien d'autres éléments comme le souffle, le sang, la moelle, etc., qui peuvent, dans chaque société, jouer un rôle particulier ${ }^{13}$.

Reprenons ici l'exemple des Bunaq : I'analyse des données permet de comprendre comment ils formalisent leurs conceptions de l'organisation du monde à travers leurs mythes et leurs rites. II s'agit d'un ensemble cohérent qui fait apparaitre deux circuits de transformation des composantes du vivant. Dans l'un,

${ }^{13}$ Dans ses travaux F. Héritier (cf. en particulier les résumés des cours de 1984 à 1990) a montre comment, dans de nombreuses sociétés, les hommes ont de tout temps établi des rapports entre la façon de s'alimenter, de se marier, d'envisager les phénomènes climatique et les conceptions qu'its ont sur le rôle des différentes humeurs do corps. En revanche peu

d'anthropologues on montré comment, dan une société particulière s'établit le cycle de transformation des composantes du vivant à travers les événements et les rituels qui marquent la vie et la mort des individus. Sur ce thème on pourra lire D. de Coppet

$(1990,1995)$

${ }^{14}$ On trouvera plus de détails sur ce cycle de transformation dans $C$. Barraud et C. Friedber

(1996).

${ }^{15}$ Le Seigneur à l'œil et à l'oreille unique est un être coupé en deux qui est célibataire et n'a pas de descendance : c'est un de enfants de l'un des couples

qui ont donné naissance aux ancêtres des Bunaq. que nous appellerons - la voie froide *, le devenir des êtres à travers le déroulement de la vie et de la trophique (comme on dirait en écologie) : les plantes sont consommées par le bétail et les hommes, le bétail est mangé par les hommes ${ }^{14}$. Ces derniers, quand ils meurent normalement, c'est-à-dire, disent les Bunaq, de vieillesse ou de maladie, voient les composantes de leur être partagées entre le corps qui est enterré et digéré par la terre et ce qui va dans le monde d'en haut. Une partie de leur âme melo he - I'âme qui vole ", est immédiatement emportée, pour être dévorée, dans la poêle à frire du Seigneur à un seul œil et à l'oreille unique ${ }^{15}$, gardien de la Maison Primordiale, source de tout ce qui existe sur terre. Un texte, récité lors des rituels qui suivent la mort, raconte comment les oiseaux, émissaires du Seigneur à l'œil unique viennent chercher le défunt. A la fin des dernières cérémonies funéraires qui peuvent se dérouler longtemps après la mort, l'autre partie de l'âme melo si, - âme chair ", qui épouse la forme du corps, rejoint le village des ancêtres, tandis qu'il y a séparation entre les * racines - du mort et celles des vivants.

La racine est en effet, aux yeux des Bunaq, un élément primordial des êtres vivants. Alors que celles des plantes sont visibles, celles des humains et des animaux ne le sont pas et des objets matériels les représentent : l'autel collectif du village pour ce qui est des personnes en tant que membres de ce village, des disques et des objets d'or et d'argent, formant ce qui est appelé le bulot, conservés dans chaque maison, pour ce qui est des personnes en tant que membres de ces maisons qui sont l'unité de base de la société. désigné comme huruk gie - appartenant au froid . mort se présente sous la forme d'une chaîne
Du côté de * la voie froide *, il y a transformation d'un état dans un autre, séparation, mais non coupure. La coupure, nous la trouvons sur l'autre circuit tinoq gie - appartenant au chaud ", celui des guerres et des males-morts, également désigné par l'expression tigi ho * le sang qui s'égoutte ", manifestation concrète de la coupure. Quand il y a malemort, aucun émissaire ne vient chercher l'âme ; c'est aux hommes de faire le rituel nécessaire pour la conduire dans la poêle du Seigneur à l'œil unique, assurant ainsi la poursuite du cycle.

Aucun texte, aucun commentaire des * maîtres de la parole ${ }^{-16}$ n'explicite ce qui se passe dans la Maison primordiale qui est à l'origine de tout ce qui existe sur terre. C'est là en particulier que le héros du mythe, racontant l'origine des plantes cultivées, est allé demander des semences aux ancêtres primordiaux. Pour que ces semences soient accessibles aux hommes, ce héros meurt, et c'est de son corps que naissent toutes les plantes cultivées. Les circonstances de cette mort ne sont pas très explicites et varient d'une version à l'autre. II s'agit soit d'un suicide, soit d'un meurtre, mais toujours le héros meurt brûlé sur l'autel qui se trouve au centre d'un champ, lors du brûlis de préparation de ce dernier. Chaque année, les semences sont accueillies sur l'autel collectif de chaque village lors de rituels précédant les semailles qui, en particulier, commémorent cette mort. Celle-ci, qui apparait clairement comme une transformation, a lieu sur la - voie froide -. Elle sera suivie par d'autres morts, celles des plantes cultivées, elles aussi destinées à être transformées en matière vivante lors de leur consommation par les humains.

Les rituels funéraires et les textes récités à cette occasion, conduisent à penser que, pour assurer la continuité du cycle vital, il est nécessaire qu'une partie des composantes du vivant revienne dans la Maison primordiale et y soit dévorée, c'est-à-dire digérée. Les unes entrent par la voie froide, les autres par la voie chaude, c'est-à-dire qu'il y a là association du froid et du chaud tout comme dans la naissance de vies nouvelles. En effet pour les Bunaq, pour qu'il $y$ ait apparition d'un nouvel être, il faut qu'il y ait association d'une semence féminine chaude et d'une masculine froide. Ainsi conçoivent-ils la réunion des semences au moment de l'acte sexuel, celles de la femme qui doivent être chaudes et celles contenues dans le sperme masculin qui est froid. Pour ce qui est des plantes, en particulier des plantes cultivées, les semences, considérées comme masculines, doivent être symboliquement refroidies avant d'être mises en terre où elles rencontreront les semences chaudes de la terre. Ces dernières sont visibles quand les premières pluies tombent sur la terre surchauffée à la fin de la saison sèche. On les voit alors s'échapper sous forme de vapeur; on dit aussi que c'est le souffle de la terre : c'est le moment de semer avant qu'elles ne s'échappent complètement.

Cependant, la nécessité d'un rituel analogue à celui que l'on effectue dans le cas de males-morts pour les humains existe aussi pour les plantes dont la vie est interrompue pour un usage autre qu'alimentaire, c'est-à-dire s'il n'y a pas transformation comme cela se produit sur la " voie froide ". Ainsi, quand on 
coupe de gros arbres pour la construction dune maison ou pour fabriquer un cercueil, il est nécessaire de tuer une chèvre au moment où l'arbre est abattu. L'âme de ce dernier est censée être transportée dans la poêle du Seigneur à l'œil unique sur les cornes de cette chèvre.

Sans doute existait-il également un rituel collectif de ce type quand l'essartage était effectué dans le cadre d'un assolement rotatif. Maintenant, le rituel n'est exécuté que Iorsqu'un bon agriculteur tombe malade : on suppose que c'est une vengeance des " mères et pères des plantes " parce que les choses n'ont pas été faites dans les règles.

Ce qui est recherché à travers ces actions rituelles est la continuité dans les processus de fonctionnement du monde. Or nous constatons que la possibilitè d'assurer une telle continuité est fondée sur l'idée qu'il existe, par delà la diversité des êtres, quelque chose qui leur est commun et qui est susceptible de se transformer, de passer d'un état à un autre, d'un règne à l'autre, englobant la mort dans le processus vital. Il ne s'agit pas ici de confusion entre les êtres, mais d'une unité qui se situe à un autre niveau que celui de l'existence de ces mêmes êtres en tant qu'individus et qui concerne les composantes du vivant. Nous sommes loin ici de la vision réductrice que l'on donne habituellement des sociétés dites * animistes *

Ce que les hommes mettent en jeu s'ils transgressent les règles rituelles, ce n'est pas leur vie en tant qu'individu, mais celle de leur société. Ces règles s'accommodent ou non de plus ou moins de diversité, de plus ou moins de discontinuité entre les êtres.

A Bali, l'ordre instauré par le rituel exige le maintien d'une discontinuité très fine entre les différents types d'êtres. Au contraire, chez les Bunaq, il existe un modèle transformationnel qui s'accommode d'un certain flou : par exemple, à l'intérieur du règne végétal, les variétés d'ignames peuvent se transformer en d'autres variétés, mais il existe aussi des passages d'un règne à l'autre dans les mythes et dans les rites.

Nous voyons donc comment à travers l'idée qu'une société se fait de l'organisation de l'ensemble des êtres vivants, on peut appréhender comment la vie individuelle est transcendée au bénéfice du fonctionnement de cette société, elle-même insérée dans le reste de l'univers. Les derniers exemples présentés ici ont été pris dans des sociétés non-modernes, mais on pourrait également en trouver dans certains aspects de nos sociétés modernes, en particulier dans la forme christianisée des rituels agraires et la façon dont il n'y a pas si longtemps, les morts étaient associés au cycle saisonnier végétal.

Les rituels apparaissent alors comme destinés à une remise en ordre perpétuelle de ce que la vie dérange de façon inévitable par son déroulement même. C'est ce qu'affirment les Balinais quand ils expliquent comment il est nécessaire d'exécuter périodiquement les cérémonies au cours desquelles on dispose sur le sol des offrandes (caru) classant différents types de nourriture et de fleurs selon les directions de l'espace, " pour remettre les choses en place ".

Cette façon de voir n'est pas très éloignée de ce que disent les scientifiques quand ils associent l'en-

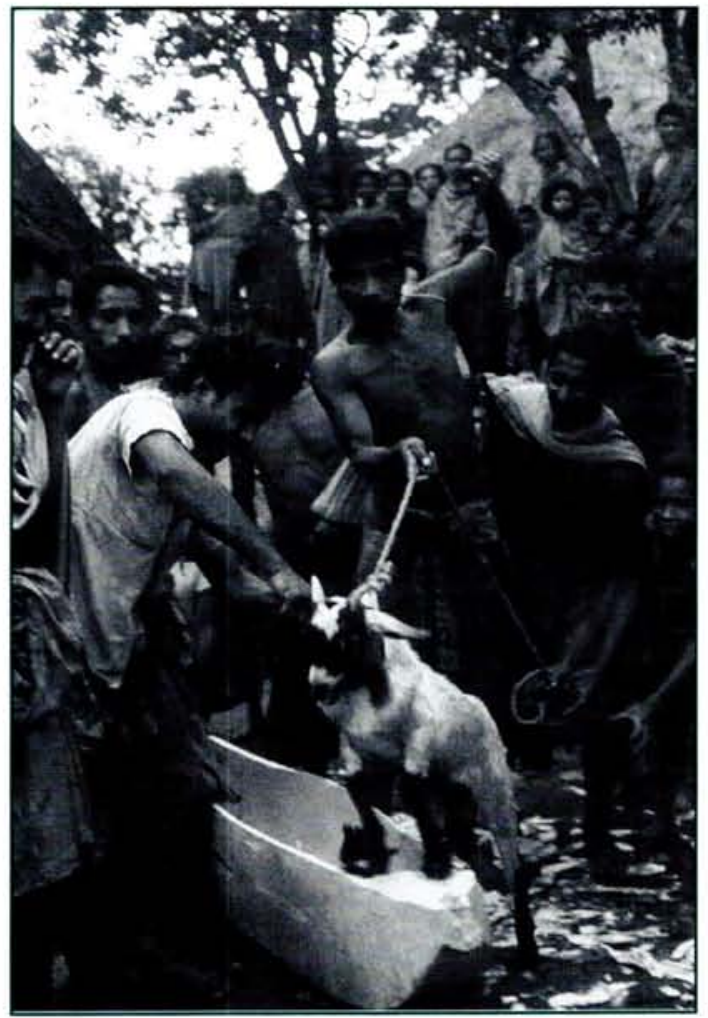

tropie maxima au désordre et à la mort et parlent comme Schrödinger (1986) de * la tendance naturelle des choses à se rapprocher de l'état de chaos à moins que nous n'y mettions obstacle - (p. 175) alors que la * propriété intrinsèque à la matière vivante est d'aller contre le désordre - (p. 23).

A Bali, les rituels évoqués ici, sont une mise en ordre perpétuellement recommencée de la diversité des êtres. Chez les Bunaq, il s'agit d'assurer la continuité du vivant sous ses diverses formes, impliquant l'idée qu'il existe une unitẻ de ce vivant au niveau de ses composantes ${ }^{17}$.

On ne peut évidemment s'empêcher de déceler ici aussi, quelque chose qui ressemble étrangement à ce que la science moderne dit sur l'unité et la continuité du vivant. La différence est que les éléments sur lesquels se construit cette unité se situent non pas au niveau des constituants les plus simples accessibles à l'expérimentation, mais à celui d'entités complexes intervenant au niveau le plus englobant de l'Univers.

\section{Conclusion}

Au terme de ce parcours rapide des différents niveaux d'organisation des savoirs sur la nature, nous pouvons mieux prendre la mesure de leur plus ou moins grande dépendance les uns vis-à-vis des autres et apprécier les divers facteurs contribuant à leur formation.
${ }^{16}$ Les lal gomo - maitres de la parole - sont les spécialistes des textes mythiques et généalogiques. Cependant toute la population bunaq a une certaine connaissance de ces textes qui sont récités en de multiples occasions rituelles. Ce sont des textes en vers formés de deux hémistiches; le lal gomo récite le premier et la foule répond par le second.

17 II ne s'agit pas de comparer ici deux sociétés différentes, ce qui dépasserait l'objectif de cet article, mais d'évoquer deux façons de traiter de lordre des choses.

Pour ce qui est de Bali, it existe bien d'autres types de rituels que ceux évoqués ici. 
Résumé : Diversité, ordre et unité du vivant dans les savoirs populaires

Au moment oủ les savoirs populaires concernant la gestion de l'environnement sont de plus en plus sollicités, il est important de comprendre comment ils s'élaborent. Pour ce qui est de l'environnement végétal et animal, il est essentiel de connaître à quel degré de diversité les organismes sont identifiés et quel type de classification leur est appliqué. L'objectif est de montrer ici comment, en prenant des exemples dans le monde végétal, les représentations de ce dernier sont liées à la fois aux caractéristiques des plantes, aux processus cognitifs propres à l'homme, et au rôle que jouent ces plantes dans la société. La reconnaissance de la diversité des êtres vivants et leur classification constituent deux niveaux d'organisation des savoirs. II en existe un troisième englobant l'ensemble des êtres vivants, y compris les êtres humains, dans le fonctionnement de chaque société. II ne s'agit plus là seulement des individus mais des éléments qui les composent et de leur devenir. Quand on veut agir sur la gestion de l'environnement, il est nécessaire de tenir compte des idées que chaque société se fait du fonctionnement du monde.

Nous avons constaté que le voir s'articule avec le faire, que le perceptuel interfère avec le conceptuel et jaugé l'importance capitale des données de l'observation. C'est à partir de ces dernières que se construisent les représentations de la nature et du fonctionnement du monde. Même si les élaborations conceptuelles influent en retour sur la perception de la réalité, il n'en demeure pas moins que les qualités intrinsèques des objets naturels ainsi que les processus cognitifs dont tout homme dispose en raison des caractéristiques génétiques de l'espèce à laquelle il appartient, jouent un rôle fondamental. Le conceptuel n'est pas créé ex nihilo à partir de l'imagination des hommes.

Les relations entre les pratiques et les représentations interviennent dans tous les aspects du processus classificatoire : identification, dénomination, insertion dans un système de référence. De ce fait les classifications sont influencées par l'évolution de ces relations dans le cadre des transformations subies par les sociétés et, en particulier, quand l'exploitation des ressources spontanées et domestiquées est modifiée par la modernisation. Inversement, l'analyse des catégories est un moyen de mettre en évidence des pratiques.

Ainsi, si la reconnaissance de la diversité et l'appréhension de la discontinuité sont conditionnées par les capacités cognitives du cerveau, elles sont également dépendantes des pratiques et contrôlées socialement.

C'est précisément dans la mesure où ils reflètent à la fois les qualités intrinsèques des objets naturels et les pratiques dont ces derniers font l'objet que les savoirs populaires présentent actuellement un intérêt pour la gestion de l'environnement. Parce qu'ils pren- nent en compte les conditions écologiques et sociales locales et leur évolution sur le long terme, ces savoirs sont dépositaires de connaissances qui exigeraient des scientifiques des observations prolongées et exhaustives incompatibles avec les moyens dont ils disposent. Pour ce qui est de la mise en évidence d'entités discrètes et de leur mise en ordre dans un système classificatoire, qui sont les deux premiers niveaux de réalité dont nous avons traité, il est possible d'établir un dialogue entre représentations scientifiques et représentations populaires en se référant à ces entités concrètes. Ce dialogue n'est pas toujours facile, il peut même être conflictuel comme nous le montre l'exemple balinais pour ce qui est des variétés de riz. La disparition des variétés nécessaires aux rituels, suite à l'imposition des riz de la révolution verte, peut déstabiliser la représentation de l'ordre du monde que les hommes s'efforcent perpétuellement de renouveler à travers les offrandes. En effet, c'est quand on parvient au troisième niveau, le niveau englobant l'ensemble du fonctionnement d'une société que le divorce entre savoir populaire et savoir scientifique apparaît le plus clairement, parce que ce qui est en cause, est la façon dont chacun conçoit son insertion dans le cadre des relations à ses semblables et dans celui des relations au monde.

Même s'ils relèvent de trois niveaux différents d'organisation de la réalité, il existe une cohérence entre tous les aspects des savoirs populaires propres à une société, cohérence garante de leur efficacité dans la logique de cette société. L'idée que l'on se fait des êtres vivants en tant qu'individus et de leur insertion dans un système organisé est subordonnée au concept d'unité et de continuité du vivant. Ceci est vrai pour les sociétés non-modernes, en particulier pour celles qui, sans accepter la modernité, accèdent de gré ou de force au modernisme, mais aussi dans notre propre société où certaines revendications écologistes montrent bien que la modernité n'atteint que certains aspects de notre existence et que la coupure nature/culture n'est pas aussi absolue que l'on voudrait le croire.

Les idées qu'une société se fait de l'organisation et du fonctionnement du monde ne sont pas toujours faciles à mettre en évidence dans la mesure où de nombreux aspects demeurent implicites, mais ce sont elles qui donnent sens aux savoirs et il est nécessaire d'en tenir compte dans l'action.

Renouer avec une conception du monde qui inclut les relations à la nature dans les pratiques sociales n'implique nullement que l'on considère les autres êtres vivants non humains comme équivalents entre eux et équivalents des hommes. Mais c'est accepter une subordination à un ordre supérieur à celui des organismes éphémères considérés comme des entités indépendantes, ordre qui conditionne la continuité du vivant. 


\section{RÉFÉRENCES}

Atran S. (1986). Fondements de I'histoire naturelle. Pour une anthropologie de la science. Paris, Complexe, $244 \mathrm{p}$.

Barraud C., Friedberg C. (1996). Life giving relationship in bunaq and kei societies, eastern Indonesia. In : For the Sake of our Future: Sacrificing in Eastern Indonesia (S. Howell J.K., ed.), Center of nonWestern Studies, Leiden

Blandin P., Arnould P. (1996). Devenir des îlots boisés dans les plaines de grande culture. Lexemple du Gâtinais nord occidental. Paris, CNRS (PIR Environnement, vie et Sociétés : comité - systèmes ruraux $\cdot$ ), 55 p (rapport final)

Buffon G.L.L. (1752). Histoire naturelle générale et particuliere avec la description du Cabinet du Roi. Paris, Imprimerie royale ( $5^{e}$ édition), vol. 1

de Coppet D. (1990). The society as an ultimate value and the sociocosmic configuration. Ethnos 55, 140-150

de Coppet D. (1995). Are'are society: a Melanesian socio-cosmic point of view. How are bigmen the servants of society and cosmos? In: Cosmos and Society in Oceania (D. de Coppet, A. Iteanu, eds), Berg Publishers Ltd, Oxford

Durkheim E., Mauss M. (1901-1902). Essais sur quelques formes primitives de classification. LAnnée sociologique 1-72

Ellen R. (1993). The cultural relations of classification. An analysis of Nuaulu animal categories from central Seram. Cambridge Studies in Social and Cultural Anthropology, Cambridge University Press, 315 p.
Friedberg C. (1990). Le savoir botanique des Bunaq. Percevoir et classer dans le haut Lamaknen (Timor, Indonésie). Mèmoires du Muséum national d'histoire naturelle, Botanique, tome 32

Friedberg C. (1996). In : - Usages des sols, représentations de la nature et dynamique des milieux et des ressources - (M Cohen, DS Lardon et al, eds), Paris, CNRS (PIR Environnement, vie et sociétés : comité - systèmes ruraux $\%, 213 \mathrm{p}$.

Hamayon R. (1990). La chasse a läme. Esquisse d'une theorie du chama. nisme sibérien. Paris, Société d'ethnologie, $879 \mathrm{p}$.

Heritier-Auge F. (1984-1990), Résumé des cours et travaux. In : Annuaire du Collège de France

Lévi-Strauss C. (1962). La Pensée sauvage. Paris, Plon, 395 p.

Lévi-Strauss C. (1982). Le Regard éloigné. Paris, Plon

Luxereau A., Roussel B. (1995). Il y a trop de gens, la terre est fatiguée : changements écologiques et changements sociaux dans la zone sahélosou. danienne du Niger central. (s.I.), (s.n.), 206 p.

Martin B., Cossalter C. (1975-1976). Les eucalyptus des iles de la Sonde. Bois et forêts des Tropiques $163,3-25 ; 164,3-14 ; 165,3-20 ; 166,3$ $22 ; 168,3-18 ; 169,3-14$

Rosch E. (1976). Classifications dobjets du monde réel : origines et représentations de la cognition. Bulletin de Psychologie, 243-249

Schrodinger E. (1986). Qu'est-ce que la vie ? De la physique à la biologie. Paris, Christian Bourgois Ėditeur (1 ${ }^{\text {re }}$ édition, 1944), $236 \mathrm{p}$.

Schrodinger E. (1992). Physique quantique et représentation du monde. Points Sciences, Le Seuil, Paris, " Science et humanisme. La physique de notre temps 